\title{
Influence of neutron transfer channels on fusion enhancement in sub-barrier region
}

\author{
Khushboo ${ }^{1, \star}$, S. Mandal ${ }^{1}$, N. Madhavan ${ }^{2}$, S. Muralithar ${ }^{2}$, J. J. Das ${ }^{2, \star \star}$, S. Nath ${ }^{2}$, A. Jhingan ${ }^{2}$, J. Gehlot ${ }^{2}$, B. Behera ${ }^{3}$, \\ S. Verma ${ }^{1}, H$. Singh $^{4}, S$. Kalkal ${ }^{1, \star \star \star}$, and $R$. Singh ${ }^{1}$ \\ ${ }^{1}$ Department of Physics and Astrophysics, University of Delhi, Delhi-110007, India \\ ${ }^{2}$ Inter University Accelerator Centre, Aruna Asaf Ali Marg, New Delhi-110067, India \\ ${ }^{3}$ Department of Physics, Panjab University, Chandigarh-160014, India \\ ${ }^{4}$ Department of Physics, Kurukshetra University, Kurukshetra-136119, India
}

\begin{abstract}
Fusion cross-section measurements were performed for system ${ }^{40} \mathrm{Ca}+{ }^{70} \mathrm{Zn}$ around the Coulomb barrier energies using Heavy Ion Reaction Analyzer (HIRA). The observed enhancement in experimental fusion cross-sections was investigated via coupled-channels formalism. The coupling of inelastic excitations alone could not reproduce the experimental data, however, the effect of octupole state of the projectile was observed to be significant. The multi-neutron positive Q-value transfer channels were also included in the calculations using semi-classical model. It was observed that two neutrons pick-up channel gave a major contribution to the fusion enhancement and successfully reproduce the experimental data at above as well as below barrier energies. The coupling of more than two neutrons transfer could not give any significant enhancement to subbarrier fusion.
\end{abstract}

\section{Introduction}

The fusion reaction is an extensively studied phenomenon at and near the Coulomb barrier. A numerous investigations were performed in past years with various systems which showed a large enhancement of fusion crosssections in comparison with theoretical predictions at subbarrier energies [1]. It was demonstrated under the framework of coupled-channels (CC) formalism that fusion is influenced by inelastic excitations of colliding nuclei and transfer of neutrons [2]. The effect of inelastic excitations were well understood with the available theoretical models. However, due to the complicated mechanism involved in coupling the transfer channels in theoretical models, the role of multi-neutron transfer on fusion was not explored in detail.

The influence of transfer on fusion was noticed in systems having a positive Q-value for neutron transfer channels [3-9]. However, in few recent work, in spite of positive Q-values, no effect of the transfer channel on fusion enhancement was observed [10-12]. Apart from this, it was demonstrated in a literature that only outermost neutrons (up to two neutrons) of nuclei give a significant contribution to the fusion enhancement [13]. The importance of two neutrons pick-up channel was also highlighted for

\footnotetext{
^e-mail: s12khushboo@gmail.com

${ }^{\star}$ Present address: National Superconducting Cyclotron Laboratory (NSCL) 640 South Shaw Lane, Michigan State University, East Lansing, Michigan-48824, USA

$\star \star \star$ Present address: School of Physics and Materials Science, Thapar University, Patiala-147004, India
}

system ${ }^{40} \mathrm{Ca}+{ }^{64} \mathrm{Ni}$ whose experimental data was well reproduced by coupling with two neutron transfer. This system has positive Q-value for up to six neutrons pick-up channels [14]. Further, the effect of pair transfer on fusion enhancement was reported recently by Stefanini et al. $[15,16]$ for ${ }^{40} \mathrm{Ca}+{ }^{96} \mathrm{Zr}$ system where the fusion crosssections were measured at deep sub-barrier energies. No fusion hindrance was observed in this system. It was pointed out that strong transfer couplings may be the reason for this observation. Beside these observations, the importance of multi-neutron transfer on sub-barrier fusion was also indicated in an article [5].

Considering all the above observations, in order to explore the effect of transfer channels on fusion enhancement, ${ }^{40} \mathrm{Ca}+{ }^{70} \mathrm{Zn}$ system was selected for the present study. ${ }^{40} \mathrm{Ca}$ is a doubly magic nucleus, however, octupole state was shown to be an important state for ${ }^{40} \mathrm{Ca}$ [17]. The system has positive Q-value for seven neutrons pick-up channels (2n-8n), therefore, importance of multi-neutron transfer on fusion can be examined with this system. The Q-values for $2 \mathrm{n}$ to $8 \mathrm{n}$ pick-up channels are $4.14,1.88$, 5.96, 2.31, 4.73, 0.14 and $0.98 \mathrm{MeV}$ respectively.

\section{Experiment Details}

The fusion cross-sections for ${ }^{40} \mathrm{Ca}+{ }^{70} \mathrm{Zn}$ were measured at Inter University Accelerator Centre (IUAC), New Delhi. A pulsed beam of ${ }^{40} \mathrm{Ca}$ was used to bombard a selfsupporting ${ }^{70} \mathrm{Zn}$ target (95\% isotopic enrichment) of 670 $\mu \mathrm{g} / \mathrm{cm}^{2}$ thickness. The measurements were carried out using the Heavy Ion Reaction Analyzer (HIRA) [18] which 
was kept at zero degree with respect to beam direction with $5 \mathrm{mSr}$ of solid angle acceptance. At the focal plane of HIRA, a Multi-Wire proportional Counter (MWPC) of area $15 \times 5 \mathrm{~cm}^{2}$ was placed followed by segmented Ionization Chamber (IC) of $7.0 \times 3.5 \mathrm{~cm}^{2}$ area and active lengths of $3 \mathrm{~cm}-5.8 \mathrm{~cm}-13 \mathrm{~cm}$ for detection of evaporation residues (ERs) arriving at the focal plane. During the experiment, MWPC and IC were operated at a pressure of $2 \mathrm{mbar}$ and $30 \mathrm{mbar}$ of isobutane gas respectively. In addition to these detectors, two Silicon Surface Barrier Detectors (SSBD) were kept symmetrically at an angle of $25^{\circ}$ on both the sides of incoming beam inside the target chamber. These detectors were used to monitor the beam during experiment and for normalization of fusion cross-sections. A carbon foil of thickness $40 \mu \mathrm{g} / \mathrm{cm}^{2}$ was placed $10 \mathrm{~cm}$ downstream from the target for equilibration of charge states of ERs. For estimation of HIRA transmission efficiency for ERs, a High Purity Germanium (HPGe) detector was mounted on top of the target chamber at $90^{\circ}$ to the beam direction.

The cross-section measurements were performed around the Coulomb barrier from laboratory energies 142 to $106 \mathrm{MeV}$. The fusion cross-sections were calculated using the yield of evaporation residues (ERs) which were separated from the beam-like particles at the focal plane using energy loss in MWPC versus time of flight (TOF) spectrum [5]. For the cross-section calculations, transmission efficiency of HIRA was estimated experimentally by gamma ray coincidence technique [5] and it was found to be $7.8 \%$ at $130 \mathrm{MeV}$ laboratory energy. It was also calculated as 7.6\% using Monte-Carlo code TERS [19].

\section{Results and Discussion}

The experimental fusion cross-sections were extracted and compared with the theoretical calculated cross-sections based on coupled-channels formalism as shown in figure 1. The experimental error in fusion cross-sections includes statistics related error and the error in transmission efficiency. The reported energies are corrected for loss in half thickness of the target. For initial estimation of theoretical fusion cross-sections, Akyuz-Winther (AW) parameterization was employed for Woods-Saxon potential. The AW parameters for the ${ }^{40} \mathrm{Ca}+{ }^{70} \mathrm{Zn}$ system were $\mathrm{V}_{0}=69.87 \mathrm{MeV}, \mathrm{r}_{0}=1.18 \mathrm{fm}$ and $\mathrm{a}_{0}=0.67 \mathrm{fm}$, which gave barrier parameters as $\mathrm{V}_{\mathrm{b}}=76.76 \mathrm{MeV}, \mathrm{R}_{\mathrm{b}}=10.48 \mathrm{fm}$ and $\hbar \omega=3.70 \mathrm{fm}$. The calculations were performed using CCFULL code [20] by considering projectile and target nuclei as a vibrator. To begin with, the coupling was switched off and no coupling fusion excitation function was obtained which underpredicted the experimental cross-sections. Therefore, in order to obtain a good fit to the above barrier experimental data, potential parameters were varied. The new set of parameters gave a slightly lower potential barrier as compared to the barrier obtained with AW parameters $\left(\mathrm{V}_{\mathrm{b}}\right)$. Using these new set of potential parameters, calculations were performed to estimate the fusion cross-sections with state $3^{-}\left(\mathrm{E}_{3^{-}}=3.74 \mathrm{MeV}\right.$, $\beta_{3}=0.33$ ) of the projectile. Coupling to this state gave a considerable enhancement to the cross-sections in the above barrier region and overpredicted the data in near barrier region. The new set of potential parameters could not give an appropriate fit to the above and near barrier data with projectile coupling hence, it was decided to use AW parameters for further calculations. After including the projectile octupole $\left(3^{-}\right)$state with $\mathrm{AW}$ parameters, the cross-sections were enhanced significantly and a reasonable fit to the above and near barrier fusion cross-sections was obtained as can be seen in figure 1. Whereas, the state $2^{+}\left(\mathrm{E}_{2^{+}}=3.90 \mathrm{MeV}, \beta_{2}=0.123\right)$ of ${ }^{40} \mathrm{Ca}$ gave a negligible contribution to the fusion cross-sections. In projectile excitations, $3^{-}$state of ${ }^{40} \mathrm{Ca}$ seems to be an important state.

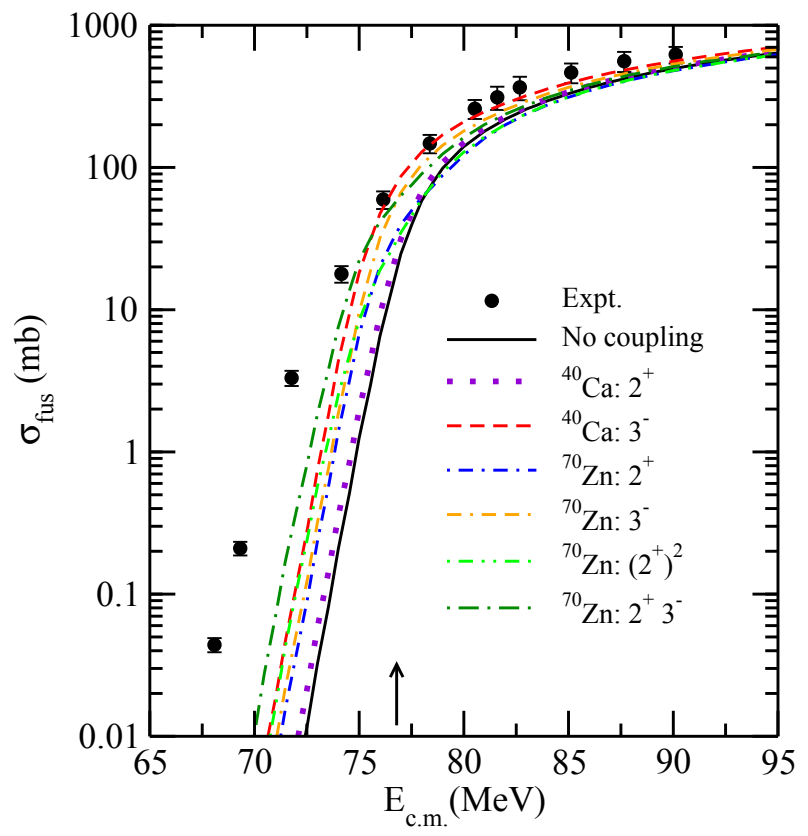

Figure 1. Experimental fusion cross-sections for ${ }^{40} \mathrm{Ca}+{ }^{70} \mathrm{Zn}$ system along with coupled-channels calculations performed with CCFULL code. The arrow at the bottom indicates the position of the barrier.

In case of target excitations, both $2^{+}\left(\mathrm{E}_{2^{+}}=0.88 \mathrm{MeV}\right.$, $\left.\beta_{2}=0.23\right)$ as well as $3^{-}\left(\mathrm{E}_{3^{-}}=2.86 \mathrm{MeV}, \beta_{3}=0.22\right)$ states enhanced the cross-sections by a similar amount in below barrier region however, $3^{-}$state gave an additional enhancement in the above barrier region. The multi-phonon and mutual excitations were also taken into consideration in the calculations. The two phonon $2^{+}$state of target further enhanced the cross-sections slightly and the excitation $2^{+} 3^{-}$gave a considerable enhancement to the crosssections. When both projectile as well target excitations were considered simultaneously in the calculations, then excitation ${ }^{40} \mathrm{Ca}: 3^{-}$and ${ }^{70} \mathrm{Zn}: 2^{+} 3^{-}$overpredicted the data in near barrier region while not explaining the data in subbarrier region. Whereas, the excitation ${ }^{40} \mathrm{Ca}: 3^{-}$and ${ }^{70} \mathrm{Zn}$ : two phonons of $2^{+}$reproduced the data reasonably well in above and near barrier energy region. This is depicted in figure 2.

The inelastic excitations alone could not reproduce the experimental data. This indicates that apart from inelastic excitations, transfer channels may play an important role for ${ }^{40} \mathrm{Ca}+{ }^{70} \mathrm{Zn}$ as Q-value is positive for multi-neutron 


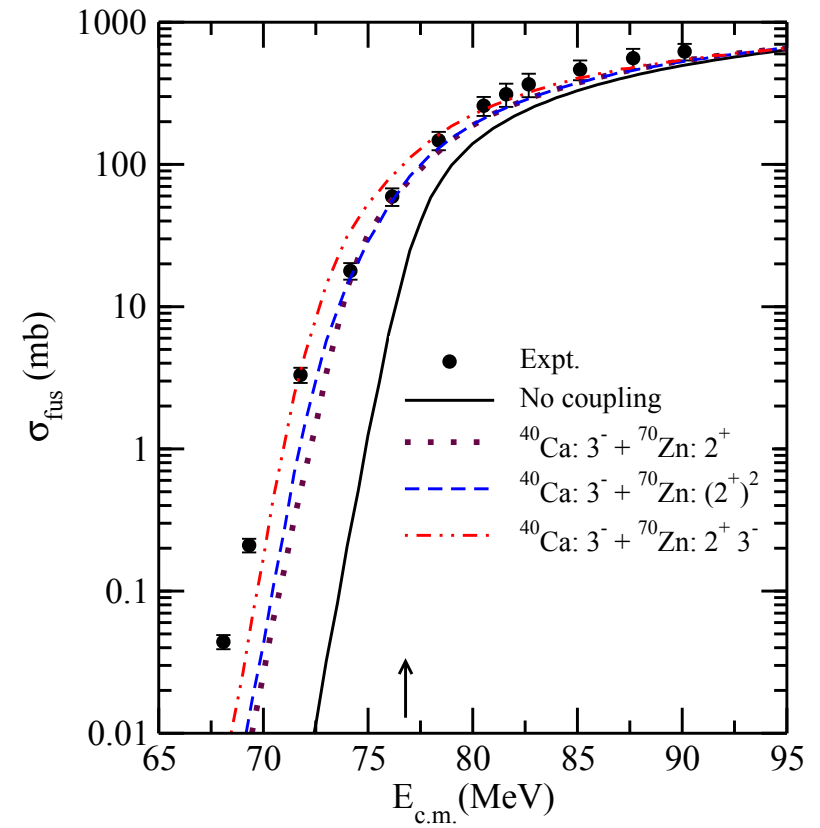

Figure 2. Experimental fusion cross-sections for ${ }^{40} \mathrm{Ca}+{ }^{70} \mathrm{Zn}$ system along with coupled-channels calculations performed with CCFULL code. The combined effect of projectile and target inelastic states are shown with different colored lines (See text). The arrow at the bottom indicates the position of the barrier.

pick-up channels. The code CCFULL considers only one pair of transfer channel between ground states. In order to examine the role of multi-neutron transfer, semiclassical model (empirical coupled-channels, ECC) of Zagrabaev et al. [21] was undertaken to perform the calculations through which one can incorporate up to four neutron transfer channels in the calculations on the basis of Q-value. Initially, the fusion excitation function was obtained without coupling of transfer channels as shown in figure 3. After that, neutron pick-up channels were successively added in the calculations. It can be seen that one neutron pick-up channel has negligible effect on fusion enhancement. The two neutron pick-up channel enhanced the sub-barrier cross-sections to a large extent and give an appropriate fit to the entire experimental data. The transfer channels $3 \mathrm{n}$ and $4 \mathrm{n}$ pick-up could not give any significant enhancement to the cross-sections which implies that the sub-barrier fusion enhancement was mainly due to the two neutron transfer despite the presence of a large number of positive Q-value transfer channels.

A detail investigation is still required to clarify the impact of two or multi-neutron transfer on fusion. The explicit coupling of multi-neutron transfer channels in the theoretical calculations will be advantageous to disentangle the neutron transfer and structure effects on fusion enhancement. The structure of the colliding nuclei may also affect the transfer coupling itself and thereby fusion. The excitation process involved during the rearrangement of nucleons between colliding partners may influence the dynamics of the sub-barrier fusion process. The presently available exotic ions from radioactive beam facilities opens up a possibility to explore the dynamics of the fusion reactions far away from the stability line where transfer and break up mechanisms may strongly influence the fusion cross-sections around the barrier and in subbarrier region.

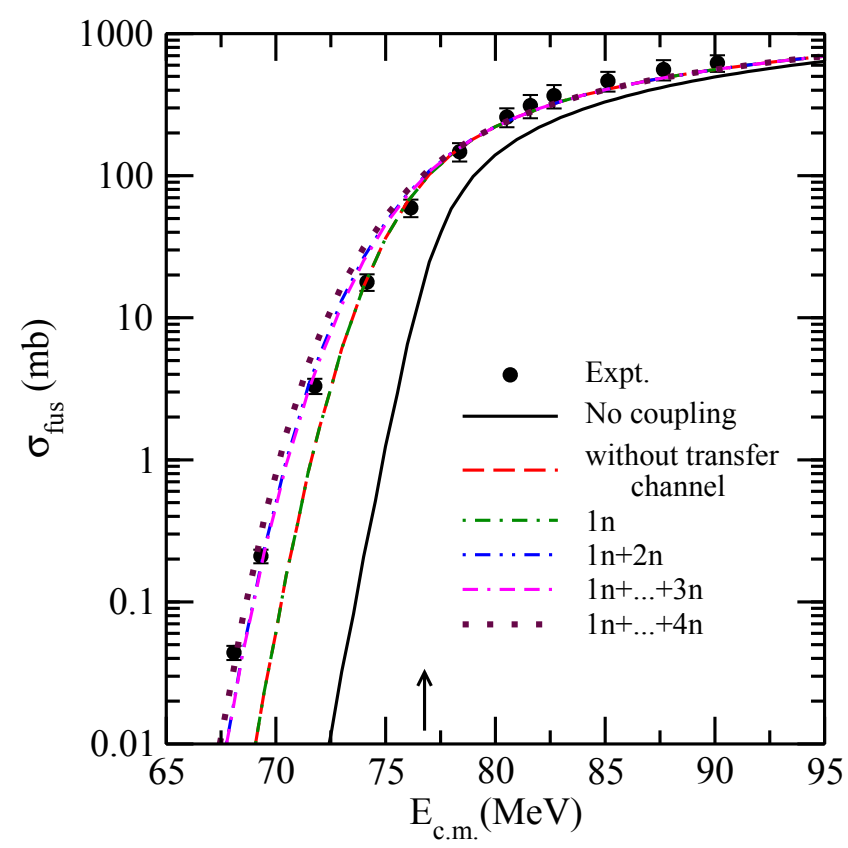

Figure 3. Experimental fusion cross-sections for ${ }^{40} \mathrm{Ca}+{ }^{70} \mathrm{Zn}$ system compared with the theoretical cross-sections obtained using ECC model. The arrow at the bottom indicates the position of the barrier.

\section{Summary}

Fusion excitation function was measured for the system ${ }^{40} \mathrm{Ca}+{ }^{70} \mathrm{Zn}$ to explore the role of multi-neutron transfer on sub-barrier fusion enhancement. The cross-sections were analyzed within coupled-channels formalism using CCFULL and ECC model. The effect of octupole vibration of ${ }^{40} \mathrm{Ca}$ was found to be major which gave a noticeable enhancement to the cross-sections in the entire energy range. The present results show the importance of transfer channels for explaining the sub-barrier fusion enhancement, however, transfer of only two neutrons was sufficient to reproduce the fusion cross-sections in above and below barrier energy region. It was observed that transfer channels beyond two neutrons have a negligible effect on subbarrier fusion enhancement indicating the less importance of large number of neutron transfer on sub-barrier fusion.

\section{Acknowledgements}

We would like to thank Pelletron group of IUAC for providing a good quality of beam throughout the experiment. The authors are extremely thankful to target laboratory of GSI and H. J. Wollersheim for providing the target. We would like to acknowledge the help received from Ranjeet during the experiment. One of the author (Khushboo) 
would like to thank SERB, Goverment of India for providing international travel grant to attend FUSION17 conference. The financial support of the IUAC research project under UFR-51314 is gratefully acknowledged.

\section{References}

[1] M. Dasgupta, D. J. Hinde, N. Rowley, and A. M. Stefanini, Annu. Rev. Nucl. Part. Sci. 48, 401 (1998)

[2] A. M. Stefanini, D. Ackerman, L. Corradi, J. H. He, G. Montagnoli, S. Beghini, F. Scarlassara, and G. F. Segato, Phys. Rev. C 52, R1727 (1995)

[3] R. A. Broglia, C. H. Dasso, S. Landowne, and A. Winther, Phys. Rev. C 27, 2433 (1983)

[4] H. Timmers, D. Ackermann, S. Beghini, L. Corradi, J. He, G. Montagnoli, E. Scarlassara, A. Stefanini, and N. Rowley, Nucl. Phys. A 633, 421 (1998)

[5] S. Kalkal, S. Mandal, N. Madhavan, E. Prasad, S. Verma, A. Jhingan, R. Sandal, S. Nath, J. Gehlot, B. R. Behera, M. Saxena, S. Goyal, D. Siwal, R. Garg, U. D. Pramanik, S. Kumar, T. Varughese, K. S. Golda, S. Muralithar, A. K. Sinha, and R. Singh, Phys. Rev. C 81, 044610 (2010)

[6] H. Q. Zhang, C. J. Lin, F. Yang, H. M. Jia, X. X. Xu, Z. D. Wu, F. Jia, S. T. Zhang, Z. H. Liu, A. Richard, and C. Beck, Phys. Rev. C 82, 054609 (2010)

[7] G. Montagnoli, A. M. Stefanini, H. Esbensen, C. L. Jiang, L. Corradi, S. Courtin, E. Fioretto, A. Goasduff, J. Grebosz, F. Haas, M. Mazzocco, C. Michelagnoli, T. Mijatovic, D. Montanari, C. Parascandolo, K. E. Rehm, F. Scarlassara, S. Szilner, X. D. Tang, and C. A. Ur, Phys. Rev. C 87, 014611 (2013)

[8] M. Trotta, A. M. Stefanini, L. Corradi, A. Gadea, F. Scarlassara, S. Beghini, and G. Montagnoli, Phys. Rev. C 65, 011601(R) (2001)

[9] G. Montagnoli, A. M. Stefanini, C. L. Jiang, H. Esbensen, L. Corradi, S. Courtin, E. Fioretto, A. Goasduff, F. Haas, A. F. Kifle, C. Michelagnoli, D. Montanari, T. Mijatovic, K. E. Rehm, R. Silvestri, P. P. Singh, F. Scarlassara, S. Szilner, X. D. Tang, and C. A. Ur, Phys. Rev. C 85, 024607 (2012)

[10] A. M. Stefanini, G. Montagnoli, F. Scarlassara, C. Jiang, H. Esbensen, E. Fioretto, L. Corradi, B. Back, C. Deibel, B. D. Giovine, J. Greene, H. Henderson, S.
Marley, M. Notani, N. Patel, K. Rehm, D. Sewerinyak, X. Tang, C.Ugalde, and S. Zhu, Eur. Phys. J. A 49, 63 (2013)

[11] Z. Kohley, J. F. Liang, D. Shapira, R. L. Varner, C. J. Gross, J. M. Allmond, A. L. Caraley, E. A. Coello, F. Favela, K. Lagergren, and P. E. Mueller, Phys. Rev. Lett. 107, 202701 (2011)

[12] H. M. Jia, C. J. Lin, F. Yang, X. X. Xu, H. Q. Zhang, Z. H. Liu, L. Yang, S. T. Zhang, P. F. Bao, and L. J. Sun, Phys. Rev. C 86, 044621 (2012)

[13] V. A. Rachkov, A. V. Karpov, A. S. Denikin, and V. I. Zagrebaev, Phys. Rev. C 90, 014614 (2014)

[14] D. Bourgin, S. Courtin, F. Haas, A. M. Stefanini, G. Montagnoli, A. Goasduff, D. Montanari, L. Corradi, E. Fioretto, J. Huiming, F. Scarlassara, N. Rowley, S. Szilner, and T. Mijatovic, Phys. Rev. C 90, 044610 (2014)

[15] A. M. Stefanini, G. Montagnoli, H. Esbensen, L. Corradi, S. Courtin, E. Fioretto, A. Goasduff, J. Grebosz, F. Haas, M. Mazzocco, C. Michelagnoli, T. Mijatovic, D. Montanari, G. Pasqualato, C. Parascandolo, F. Scarlassara, E. Strano, S. Szilner, D. Torresi, Phys. Lett. B 728, 639 (2014)

[16] A. M. Stefanini, G. Montagnoli, H. Esbensen, L. Corradi, S. Courtin, E. Fioretto, A. Goasduff, J. Grebosz, F. Haas, M. Mazzocco, C. Michelagnoli, T. Mijatovic, D. Montanari, G. Pasqualato, C. Parascandolo, F. Scarlassara, E. Strano, S. Szilner, N.Toniolo, D. Torresi, EPJ Web of Conferences 86, 00056 (2015)

[17] J. F. Liang, J. M. Allmond, C. J. Gross, P. E. Mueller, D. Shapira, R. L. Varner, M. Dasgupta, D. J. Hinde, C. Simenel, E. Williams, K. Vo-Phuoc, M. L. Brown, I. P. Carter, M. Evers, D. H. Luong, T. Ebadi, and A. Wakhle, Phys. Rev. C 94, 024616 (2016)

[18] A. K. Sinha, N. Madhavan, J. . Das, P. Sugathan, D. Kataria, A. Patro, and G. Mehta, Nucl. Inst. Meth. A 339, 543 (1994)

[19] S. Nath, Comput. Phys. Commun. 180, 2392 (2009)

[20] K. Hagino, N. Rowley, and A. T. Kruppa, Comput. Phys. Commun. 123, 143 (1999)

[21] V. I. Zagrebaev, A. S. Denikin, A. V. Karpov, A. P. Alekseev, M. A. Naumenko, V. A. Rachkov, V. V. Samarin, V. V. Saiko, NRV web knowledge base on low-energy nuclear physics, http://nrv.jinr.ru/. 